Aves en un humedal boscoso costero de la región de la Araucanía: Humedal de Mahuidanche

\author{
Luciano Parra-Coloma ${ }^{1}$, Mauricio Urra ${ }^{2}$, María Fernanda Aguayo-Molina ${ }^{3}$ \\ ${ }^{1}$ Cosmovitalis Consultores Ltda., Máximo Reyes 1250, Temuco, Chile. Email: \\ $\underline{\text { lucianoparrac@gmail.com }}$
}

${ }^{2}$ Profesional independiente. Email: mauricio.urra.a@gmail.com

${ }^{3}$ Profesional independiente. Email: mfaguayo.molina@gmail.com

Los humedales son considerados los ecosistemas de mayor riqueza biológica del planeta, destacándose por su gran productividad y biodiversidad (Kusler et al. 1994; Innis et al. 2000), los cuales otorgan variadas funciones como la contribución en los ciclos de vida de plantas y animales, hábitat, alimento, sitios de nidificación y refugio para numerosas especies de animales (insectos, peces, batracios, crustáceos, aves, etc.) (Perotti et al, 2005; Correa-Araneda et al. 2011).

En la región de la Araucanía presenta abundantes humedales ubicados en zonas montañosas y costeras (Hauenstein et al. 2002; De los Ríos et al. 2007), en estos últimos se desarrollan los humedales boscosos, ecosistemas de gran importancia mundial, pero poco conocidos en Chile en cuanto a su funcionamiento hidrológico y a sus efectos antropogénicos (Correa-Araneda et al. 2011). En estos humedales se destacan las asociaciones vegetacionales de Temu (Blepharocalyx cruckshanksii) y Pitra (Myrceugenia 
exsucca), en especial en el sector Mahuidanchi-Lastarria (Hauenstein et al. 2014), sin embargo, los estudios realizados en estos tipos de ecosistemas van desde un punto de vista vegetacional y florístico, careciendo información relacionada a lo faunístico (CorreaAraneda et al. 2011). En el presente trabajo indica la diversidad de aves presentes en el humedal boscoso costero, presente en la comuna de Gorbea, región de la Araucanía, Chile. El área de estudio corresponde al humedal de Mahuidanchi $\left(39^{\circ} 15^{\prime} \mathrm{S}-72^{\circ} 47^{\prime} \mathrm{W}\right)$, uno de los principales humedales boscosos presentes en la región (Correa-Araneda et al 2011). Tiene una cuenca de 2.824 hectáreas rodeada mayoritariamente por plantaciones forestales de especies exóticas de Pinus radiata y Eucaliptus globulus (Parra-Coloma y CorreaAraneda 2014). Se registraron las aves durante 3 salidas a terreno en primavera y verano durante los años 2011 y 2012, siguiendo la técnica de transecto en franjas (Ralph et al. 1996). Los registros se realizaron con binoculares $12 \times 50$ en un camino ripiado y en línea recta de 800 m aprox, en la cual no se prestó atención a la configuración de los distintos hábitats. Las descripciones de aves siguieron las descripciones de Araya y Millie (2005) y Jaramillo (2005).

El territorio nacional presenta un total de 462 especies de aves (Araya y Millie 2005), del cual el 7,14\% se encuentra representado en el humedal. Esto corresponde a un total de 33 especies y 23 familias, siendo las más dominantes Icteridae, Furnariidae, Rhynocryptidae y Tyrannidae, con 3 especies cada una (Tabla 1). De todas las especies registradas, solo Speculanas specularis se encuentra en categoría de conservación de casi amenazado según UICN. Las avifauna presente en el humedal se destaca por su alta diversidad, la cual se asocia de manera directa con la productividad de estos ecosistemas (Schlatter y Sielfeld 2006). No se aprecia un marcado endemismo en las especies que 
habitan en el humedal, ya que existen especies migratorias, como Elaenia albiceps chilensis y Tachycineta meyeni. La composición de las aves es un componente importante para la evaluación turística en humedales, ya que incluye parámetros como abundancia, valor estético, perceptibilidad, estado de conservación, endemismo, valor histórico/cultural, valor de uso e importancia científica y singularidad taxonómica (Muñoz-Pedreros \& Quintana 2010), sin embargo existe confusión y pocos estudios relacionados con los patrones de distribución y abundancia de aves en ecosistemas acuáticos (Victoriano et al 2006; Vilina y Cofré 2008). En este contexto, algunas de las aves fueron registradas en otros humedales del sur de Chile, como el humedal del río Cruces, las cuales pueden ser utilizadas como parte del turismo de especiales (Muñoz-Pedreros y Quintanilla 2010). 


\section{Tabla 1.}

Lista de aves presentes en el humedal. Familias y especies modificados de Araya \& Millie 2005 y Jaramillo (2005).

\begin{tabular}{|c|c|c|c|}
\hline Familia/Especie & Nombre común & Distribución & $\begin{array}{c}\text { Estado de } \\
\text { conservación* }\end{array}$ \\
\hline \multicolumn{4}{|l|}{ Accipitridae } \\
\hline Parabuteo unicinctus & Peuco & $\mathrm{I}-\mathrm{XI}$ & $\mathrm{LC}$ \\
\hline \multicolumn{4}{|l|}{ Anatidae } \\
\hline Speculanas specularis & Pato anteojillo & V-XII & NT \\
\hline Anas geórgica & Pato jergón grande & I-XII & $\mathrm{LC}$ \\
\hline \multicolumn{4}{|l|}{ Ardeidae } \\
\hline Ardea alba & Garza grande & I-XII & $\mathrm{LC}$ \\
\hline \multicolumn{4}{|l|}{ Cathartidae } \\
\hline Coragyps atratus & Jote cabeza negra & $\mathrm{I}-\mathrm{XI}$ & $\mathrm{LC}$ \\
\hline \multicolumn{4}{|l|}{ Charadriidae } \\
\hline Vanellus chilensis chilensis & Queltehue & III-X & $\mathrm{LC}$ \\
\hline \multicolumn{4}{|l|}{ Columbidae } \\
\hline Patagioenas araucana & Torcaza & III-XI & $\mathrm{LC}$ \\
\hline \multicolumn{4}{|l|}{ Emberizidae } \\
\hline Zonotrichia capensis chilensis & Chincol & III-XI & $\mathrm{LC}$ \\
\hline \multicolumn{4}{|l|}{ Passeridae } \\
\hline Passer domestixcus & Gorrión & I-XII & $\mathrm{LC}$ \\
\hline \multicolumn{4}{|l|}{ Icteridae } \\
\hline Sturnella loyca & Loica & III-XII & $\mathrm{LC}$ \\
\hline Curaeus curaeus curaeus & Tordo & III-XII & $\mathrm{LC}$ \\
\hline Agelasticus thilius thilius & Trile & III-X & $\mathrm{LC}$ \\
\hline \multicolumn{4}{|l|}{ Falconidae } \\
\hline Phalcoboenus chimango & Tiuque & I-XII & $\mathrm{LC}$ \\
\hline \multicolumn{4}{|l|}{ Emberizidae } \\
\hline Phrygilus patagonicus & Cometocino patagónico & V-XII & $\mathrm{LC}$ \\
\hline Carduelis barbata & Jilguero & III-XII & $\mathrm{LC}$ \\
\hline \multicolumn{4}{|l|}{ Furnariidae } \\
\hline Cinclodes patagonicus chilensis & Churrete & V-XI & $\mathrm{LC}$ \\
\hline Aphrastura spinicauda spinicauda & Rayadito & IV-XII & $\mathrm{LC}$ \\
\hline Sylviorthorhynchus desmursii & Colilarga & V-XII & $\mathrm{LC}$ \\
\hline \multicolumn{4}{|l|}{ Hirundinidae } \\
\hline Tachycineta meyeni & Golondrina chilena & III-XII & $\mathrm{LC}$ \\
\hline
\end{tabular}


Sustainability, Agri, Food and Environmental Research, 2014, 2(3): 67-73

ISSN: 0719-3726

$\begin{array}{lll}\text { Mimus thenca } & \text { Tenca } & \text { III-XIV }\end{array}$

Phalacrocoracidae

$\begin{array}{llll}\text { Phalacrocorax brasilianusbrasilianus } & \text { Yeco I-XII } & \text { LC }\end{array}$

Picidae

$\begin{array}{llll}\text { Colaptes pitius pitius } & \text { Pitío } & \text { III-X } & \text { LC }\end{array}$

Psittacidae

Enicognathus leptorhynchus Choroy V-XI LC

Rallidae

$\begin{array}{llll}\text { Pardirallus sanguinolentus landbecki Pidén I-XI } & \text { LC }\end{array}$

Rhynocryptidae

Scelorchilus rubecula rubecula

VI-XI LC

Scytalopus magellanicus magellanicus

Pteroptochos tarnii

Churrín del sur

VIII-XII

$\mathrm{LC}$

Hued-hued del sur

VII-XII

$\mathrm{LC}$

Trochilidae

Sephanoides sephanoides

Picaflor

III-XII

LC

Troglodytidae

Troglodytes aedon chilensis

Chercán

III - XII

LC

Turdidae

Turdus falcklandii magellanicus

Zorzal

III-XII

LC

Tyrannidae

Xolmis pyrope pyrope

Diucón

III-XII

LC

Elaenia albiceps chilensis

Fio Fío

III-XII

LC

Colorhamphus parvirostris

Viudita

IV-XII

LC

*Estado de conservación según UICN y el Ministerio del Medio Ambiente. 


\section{Referencias}

Araya, B. y Millie, G. 2005. Guía de campo de las aves de Chile, 9a edición. Editorial Universitaria, Santiago, Chile. 406 pp.

Correa-Araneda, F., J. Urrutia, y R. Figueroa, 2011. Estado del conocimiento y principales amenazas de los humedales boscosos de agua dulce de Chile. Revista Chilena de Historia Natural 84: 325-340

De los Ríos P., E. Hauenstein, ,P. Acevedo, y X. Jaque, 2007. Littoral crustaceans in mountain lakes of Huerquehue National Park ( $38^{\circ} \mathrm{S}$, Araucania región, Chile). Crustaceana, 80(4): 401-410.

Hauenstein, E., M. González,., F. Peña-Cortés, y A. Muñoz-Pedreros, 2002. Clasificación y caracterización de la flora y vegetación de los humedales de la costa del Toltén (IX región, Chile). Gayana Botánica 59: 87-100.

Hauenstein, E., F. Peña-Cortés, C. Beltrán, J. Tapia, L.Vargas-Chacoff, y O. Urrutia, 2014. Composición florística y evaluación de la degradación del bosque pantanosos costero de temu-pitra en la región de la Araucanía, Chile. Gayana Botánica 71(1): 43-57.

Innis, S.A., R.J. Naiman, y S.R. Elliott, 2000. Indicators and assessment methods for measuring the ecological integrity of semi-aquatic terrestrial environments. Hydrobiologia 422/423: 111-131.

Jaramillo A. 2005. Aves de Chile. Editorial Lynx, Santiago de Chile, 240 p.

Kusler, J.A., W.J. Mitsch, y J.S. Larson, 1994. Humedales. Investigación y Ciencia 210: 613. 
Muñoz-Pedreros, A. y J. Quintana, 2010. Evaluación de fauna silvestre para uso ecoturístico en humedales del río Cruces, sitio Ramsar de Chile. Interciencia 35(10): 730738.

Parra-Coloma, L. y F. Correa-Araneda, 2014. Presencia de Gambusia affinis (Baird \& Girard, 1853) en un humedal boscoso del sur de Chile. Posibles implicancias sobre la fauna local de vertebrados. Sustainability, Agri, Food and Environmental Research 2(1): 85-89. Perotti, M.G., M.C. Diéguez, y F.G. Jara, 2005. Estado de conocimiento de humedales del norte patagónico (Argentina): aspectos relevantes e importancia para la conservación de la biodiversidad regional. Revista chilena de Historia Natural 78: 723-737.

Ralph, C.J., G.R. Geupel, P. Pyle, T.E. Martin, D.F. Desante, y B. Milá, 1996. Manual de métodos de campo para el monitoreo de aves terrestres. Gen. Tech. Rep. PSW-GTR-159. Albany,CA: Pacific Southwest Research Station, Forest Service, U.S. Department of Agriculture. 46 pp.

Schlatter, R. y W. Sielfeld, 2006. Avifauna y mamíferos acuáticos de humedales de Chile. En: Vila, I., Veloso, A., Schlatter, R., \& Ramírez, C. (eds) Macrófitas y vertebrados de los sistemas límnicos de Chile. Editorial Universitaria. Santiago, Chile. 141-186 pp.

Victoriano, P.F., González, A.L. \& Schlatter, R. 2006. Estado de conocimiento de las aves de aguas continentales de Chile. Gayana 70(1): 140-162.

Vilina, Y. y H. Cofré, 2008. Aves acuáticas continentales. En: CONAMA (eds) Biodiversidad de Chile: Patrimonio y desafíos. Ocho Libros Editores, Santiago, Chile. 266273 pp. 